\title{
Effects of response type on coordinated responses during arm movement
}

\author{
RAYNA AZUMA and PATRICK HAGGARD \\ University College London, London, England
}

\begin{abstract}
This paper reports some experimental results on the coordination of finger and vocal responses with passing through a target position in multijoint arm movement. In Experiment 1, we found that the difference in the timing of finger and vocal responses cannot be attributed entirely to efferent or representational effects. Instead, it appears to reflect the extent to which information about the internal stimuli generated by the arm movement are available to the centers controlling these different responses. That is, it is a compatibility effect. In Experiment 2, the case in which a finger response is made on the same side of the body as the moving arm was compared with the case in which it is made with the contralateral hand, which remains static. The interaction effect observed suggests that the pathways subserving coordinated responses are informationally encapsulated, so that information about arm movement is not shared between the neural centers controlling different coordinated responses.
\end{abstract}

In everyday life, most human actions consist of a coordinated movement of several joints. For example, in order to grasp an object on the table, one must open the fingers before reaching the object, so as not to knock it down. Although one can open one's fingers well in advance in the case of grasping, sometimes the timing must be extremely accurate, as when throwing a ball. How do people accurately time the actions of more than one effector? Do some effectors "go together" better than others, and do such motor compatibility effects form the basis of motor skill? This paper investigates this issue in the context of coordinating discrete secondary responses with a primary arm movement.

\section{Response Timing Errors}

In a study by Haggard, Lyon, Bradbury, and Azuma (1999), subjects moved a joystick from side to side, making an arm movement across the body of around $80 \mathrm{~cm}$ amplitude. Although that study was primarily concerned with the effects of vision of the moving limb on the timing of coordinated responses, a significant main effect of response type was also observed. In brief, when subjects attempted to synchronize finger extensions with passing through the target, they actually responded $43 \mathrm{msec}$ early. However, when they attempted to synchronize a vocal response with passing the target, they actually responded $39 \mathrm{msec}$ late. The aim of this paper is to investigate at what stage of processing, from the monitoring of the moving limb to the dispatching of efferent commands for the coordinated response, these response effects may arise. In particular, are such response effects merely differences

Correspondence concerning this article should be addressed to P. Haggard, Department of Psychology, University College London, Gower St., London WCIE 6BT, England (e-mail: p.haggard@ucl.ac.uk).

-Accepted by previous editor. Mron L. Braunstein in the latencies of the neural pathways of the different response systems, or do they reflect central differences in the information processing for coordinated movement.

Most previous studies of errors in response timing have involved external synchronization tasks, in which subjects tap in time to a repetitive external stimulus, typically a metronome. This task differs from a movement coordination task in two important respects. First, synchronization involves monitoring external stimuli, whereas coordination involves monitoring the internal stimuli generated by the moving arm. Second, the stimulus in synchronization tasks forms a regular repeating pattern, whereas that in coordination tasks is a continuous varying quantity, which is independent on each successive movement trial. Despite these differences, the external synchronization literature raises several issues in response timing that may be relevant to performance on movement coordination tasks.

One of the most interesting and well-studied phenomena in the synchronization studies is negative asynchrony. Subjects tend to anticipate the auditory stimulus by a few tens of milliseconds (Aschersleben, 1994; Aschersleben \& Prinz, 1995; Dunlop, 1910; Fraisse, 1974; Vos \& Helsper, 1992; Vos, Mates, \& von Kruysbergen, 1995). Can this phenomenon explain the anticipatory nature of some responses in coordinated action also? There are two accounts of negative asynchrony that may also be relevant to movement coordination tasks. These are (1) the PaillardFraisse hypothesis and (2) the P-center hypothesis.

First, according to the Paillard-Fraisse hypothesis (Fraisse, 1974), the brain tries to synchronize sensory codes based on afferent information about events, rather than on actual onsets of the physical events themselves. In the case of tapping to a metronome, transmission of the feedback from a tap to the brain takes longer than the transmission of the auditory stimulus, largely because of the greater neural transmission distance in the former case. In order to synchronize the two sensory codes arriv- 
ing at the brain, the tap must precede the stimulus by the same amount of time as the difference in transmission times. If this hypothesis is correct, one would expect the size of tapping asynchrony to depend on the distance between the peripheral effector and the brain. Aschersleben and Prinz (1995) found a greater asynchrony for toe responses than for finger responses, which supports this view.

Second, the P-center hypothesis claims that the perceived onset (referred to as the perceptual center or $P$ center) of an event may not coincide with its physical onset. Instead, the perceived onset time is influenced by other properties of the event, such as its duration and the abruptness of its onset (Morton, Marcus, \& Frankish, 1976; Vos et al.,1995). For example, metronome clicks used in synchronization tasks may be brief, with abrupt onsets, whereas tapping movements may have a longer duration and a less abrupt onset. The P-center of the taps might thus lag their physical onset substantially, whereas this lag would be much reduced for the auditory stimuli. Subjects would thus need to tap with negative asynchrony to synchronize the P-centers of taps and clicks. This hypothesis implies that the size of the asynchrony should be positively correlated with response (tap) duration, as well as negatively correlated with stimulus (metronome) duration. Vos et al. tested the latter hypothesis by manipulating the duration of auditory stimuli and interstimulus intervals (ISIs). They found that the negative asynchrony was reduced significantly with longer stimulus durations but was not reliably affected by the ISI.

\section{Response Effects in Movement Coordination}

Both the Paillard-Fraisse account and the P-center account could also be applied to the timing of responses coordinated with primary movement. For example, differences in timing of coordinated finger and vocal responses might reflect different afferent feedback delays or different perceptual centers for these response types. Three further possibilities could also contribute to differences in response timing in coordination. We shall call these (1) the efferent account, (2) the awareness account, and (3) the availability or stimulus-response (S-R) compatibility account.

The efferent account attributes response effects solely to differences in the time taken for the efferent signal to produce movement (Anson, 1982). If the motor delay between a central command and a finger movement were shorter than the motor delay between a central command and the acoustic signal of a vocal response, the response effect observed by Haggard et al. (1999) would be obtained. However, this explanation seems unlikely, because the distance from brain to hand exceeds that to the larynx, implying that finger responses should lag vocal responses. Different inertias of the musculature of the finger and larynx might, however, mean that neural transmission time is only a small part of the total response time.

The timing of a coordinated response may also depend on whether that response is mediated by conscious aware- ness. External stimuli may be processed with or without awareness (Weiskrantz, 1974). Furthermore, motor responses to a stimulus may be dissociated from perceptual awareness of the stimulus (Bridgeman, Peery, \& Anand, 1997; Goodale, Milner, Jakobson, \& Carey, 1991a, 1991b; Goodale, Pelisson, \& Prablanc, 1986). Moreover, Castiello, Paulignan, and Jeannerod (1991) have argued that responses that are mediated by awareness of an external stimulus appear to take longer than responses that are not mediated by awareness. Those authors found that a vocal response to a sudden displacement of a visual target during a reaching movement required nearly $400 \mathrm{msec}$, whereas motor adjustment of the reaching trajectory required only $100 \mathrm{msec}$. They suggested that vocal responses had to be mediated by perceptual awareness, which involved longer processing times. Nevertheless, both methodological and theoretical problems exist for this view. It is difficult to measure awareness objectively and, thus, hard to provide independent evidence that subjects were more aware of a stimulus when they made vocal responses to it than when they made motor adjustments to it. Furthermore, there is no a priori reason to associate vocal fold movement with awareness any more than other movement.

In all of the above studies, awareness of external stimuli was investigated. How this tendency for awareness to delay responses (Castiello \& Jeannerod, 1991; Castiello et al., 1991) could be generalized to a coordination task, in which the internal stimuli generated by the moving arm must be monitored for the purposes of making a coordinated response, remains unclear. Nevertheless, experimental evidence that different responses produce different levels of awareness of the primary arm movement would permit an awareness explanation of response effects.

The availability or S-R compatibility account claims that some response centers have better access than others to the stimulus information. It is well known that some input-output mappings, such as the combination of a tactile stimulus with a manual response, or the combination of auditory input with speech output, produce better performance than others (Greenwald, 1972; Greenwald \& Shulman, 1973; Leonard, 1959; see Proctor \& Reeve, 1990 , for a review). This may arise when there is a special S-R mapping that "has a close matching between the input and output modality, to the extent that the stimulus bears a near-resemblance to the feedback produced by the response" (Barber, 1988, p. 184). If the primary movement in a coordination task can be considered to generate internal stimuli, a similar explanation can be given for response effects in coordination: Perhaps the center controlling finger responses has different access to the stimuli generated by the moving arm than does the center controlling vocal responses.

If the S-R compatibility account is correct, it is important to understand what sources of information about multijoint arm movement exist and to assess why the internal stimuli may be differentially available to different response centers. There are three main ways that a multi- 
joint movement sequence might be coordinated: motor programs, proprioception, and efference copy. The importance of the contribution that each makes is not clear.

A motor program is a package of motor commands prepared in advance. These may be run off in an openloop manner, or they may require appropriate afferent feedback for their execution (Schmidt, 1988). An influential piece of evidence for the existence of motor programs comes from reaction time (RT) studies in which response latency is observed to be longer for more complex movements, suggesting that programming takes place prior to action (Henry \& Rogers, 1960). Two movements could be coordinated by ensuring common timing of their motor programs (Jeannerod, 1981).

Proprioception offers a second source of information for coordinating multijoint movements. Several structures, such as muscle spindles and receptors in the joint capsule, can provide information about limb position and velocity, and cutaneous receptors may also contribute. The importance of the contribution that each of these sensory sources makes to kinaesthesis is unclear.

Cordo (1990) suggested that proprioceptive input related to the active rotation of a joint might be useful for triggering movement at another joint during movement sequences. On the basis of those assumptions, he ran a series of experiments in order to investigate whether the central nervous system (CNS) can use kinaesthetic input to coordinate multijoint movement sequences (Cordo, 1990; Cordo, Carlton, Bevan, Carlton, \& Kerr, 1994; Cordo \& Flanders, 1989). In the Cordo et al. experiment, the elbow was extended passively, and the subject opened the hand as the arm passed through a target zone. A hydraulic apparatus then simulated a spring resistance to the elbow extension. Since there were unexpected changes in spring constant to prevent the use of a motor program in stretching the hand to open at a fixed delay after elbow movement began and no visual feedback was available, subjects had to use kinaesthetic input to control the motor task. The significant modulation of hand opening latency observed as the time of reaching the target varied was attributed to the use of kinaesthetic information about elbow rotation in coordinating hand opening. Since hand opening sometimes preceded reaching the target zone, this kinaesthetic information must be at least partly predictive. Cordo et al. have proposed that muscle spindle signals encoding movement velocity may play a key role in this prediction.

The third possible source of information about limb position for use in coordination is efference copy. This is a putative copy of the descending motor command. It has been suggested that efference copy may underlie a sense of effort. Gandevia (1987; Gandevia \& Burke, 1992) has suggested that perceived motor commands influence the sensation of muscle force and the timing of muscular contraction. If efference copy provides a sense of the amplitude and duration of muscular activity, the CNS would use an internal model of the arm's dynamics to obtain estimates of the position and velocity of the limb that the current motor command would produce. The cerebellum is thought to do precisely this (Stein \& Glicksteith, 1992).

\section{EXPERIMENT 1}

In Experiment 1, we compared the timing of vocal responses ("Aah!"), finger responses (extension of the right index to release a pressure pad), or both of these responses made together in coordination with an arm movement. The measurement of responses is discussed in detail later. The study focused on differences between vocal and finger responses to the internal stimuli generated by arm movement. The purpose of the experiment was to distinguish between three possible sources of response effects. These were (1) differences in the quantity or quality of information about arm movement available to the finger and vocal response centers (availability or compatibility hypothesis); (2) differences in the speed of operation of the response pathways themselves (efferent hypothesis); or (3) differences in the internally represented time of the responses (e.g., P-center hypothesis). In Experiment 1, response effects were investigated in a coordination task and in control conditions of visual simple RT and self-paced synchronization tasks to investigate the origin of response effects in coordination. Response effects in the visual simple RT condition are presumed to arise from efferent factors alone. Thus, if the efferent hypothesis is correct, response effects should be comparable in coordination and in RT tasks. Response effects in the self-paced synchronization task are presumed to arise from differences in the P-centers of the two responses. Thus, if the P-center hypothesis is correct, response effects should be comparable in synchronization and RT tasks.

In Experiment 1, we also investigated whether performance of one of these responses influenced performance of the other, by including a dual-response condition in both coordination and RT tasks. Dual-task performance offers a particularly useful paradigm for investigating the availability hypothesis. If these response effects remain the same even when both responses are made on a single trial, the $S-R$ pathways subserving the two responses must be separate within the CNS and informationally encapsulated. If, on the other hand, one response attracts the other (Duncan, 1979), the neural pathway that produces the attracted response may be influenced by information in the pathway that produces the dominant (attracting) response.

\section{Method}

The basic design of the experiment was a $2 \times 2 \times 2$ factorial design. The independent variables were task (simple RT, coordination), response type (finger vs. vocal), and response number (single vs. dual). For the single-response condition either finger or vocal responses were used, whereas both responses were made within a single trial for the dual-response condition. The subjects were randomly assigned to four groups in order to counterbalance the order of the task and response type conditions. Half the subjects did the coordination task before the RT task; this order was reversed for the 
other half. Within each task, half the subjects made finger responses first, followed by vocal responses; this order was reversed for the other half. However, all the subjects performed the dualresponse condition last in each task block, to prevent any possible cooperation or information sharing between the responses from producing learning effects that might bias single-response performance. Task, response type, and response number were withinsubjects factors. Response delay in milliseconds was used as the dependent variable for most analyses. For RT tasks, the RT can be considered as a delay calculated from stimulus onset. For the coordination task, delay was calculated from the time of passing through the target (and could thus take negative values in the case of anticipatory responses).

Finally, all the subjects performed a self-paced synchronization task as a control task prior to the main experiment. This task required subjects to synchronize the onsets of vocal and finger responses (see below).

Subjects. Twelve normal subjects, of whom 5 were male and 7 were female, participated in the experiment. All were strongly right-handed, according to the Edinburgh Handedness Inventory (Oldfield, 1971). All the subjects were recruited from students currently studying at the University of London, with the exception of 1 subject, who was in employment. Their age ranged from 20 to 45 years (average, 28 years). They were paid for their contribution. All were naive as to the purpose of the experiment.

Apparatus. A joystick was used to measure the arm movement component of the coordination task. This consisted of a piece of light metal tubing, approximately $1 \mathrm{~m}$ in length, attached to a potentiometer. The movement of the joystick was restricted to an arc of approximately $80^{\circ}$, which gave its tip a tangential excursion of about $0.8 \mathrm{~m}$ (Figure 1). The potentiometer voltage was fed to a computer data acquisition system, which sampled it at $500 \mathrm{~Hz}$ and converted it into digital form to record the participant's arm movement. Movement initiation was detected by the joystick's departure from a pressure pad attached at the right limit of its movement. This event triggered the computer to acquire data. A target consisting of a vertical strip of colored paper $1 \mathrm{~cm}$ wide was positioned vertically so that the joystick passed directly in front of it, at about the center of its travel.

Finger responses were measured with a second pressure pad (Force Sensing Resistor, Interlink Electronics) mounted on the joystick handle beneath the pad of the subject's right index finger. Extension of the index finger released the pressure pad, causing a sudden drop in recorded voltage. A threshold voltage value for release of the pressure pad was chosen on the basis of comparison with a strain-gauge.

For the vocal response, the subject wore a throat microphone. The output of the microphone was fed to a voice key, which produced a digital trigger signal when sufficient acoustic energy was detected. The trigger signal occurred less than $1 \mathrm{msec}$ after the first visible sign of modulation in the acoustic signal on 10 calibration trials.

For the simple RT task, a red light-emitting diode (LED) was attached to the wall at a height of $0.8 \mathrm{~m}$. The delay between the initiation of a trial by the experimenter and the illumination of the LED was varied randomly within a range from 1,000 to $2,000 \mathrm{msec}$. Vocal RTs were recorded as in the coordination task. For the finger response, the subject extended his or her index finger so as to release a pressure pad attached to a $10-\mathrm{cm}^{2}$ tile, placed on his or her knee.

Protocol and instructions. The subjects were tested individually. Instructions were given before each task.

For the self-paced synchronization task, the subjects were asked to use a pressure pad, mounted on a tile on their knees, and the throat microphone. For the finger response, they were told that they had to maintain a firm pressure on the pad with the right index finger and then release it by a sudden, sharp movement. For the vocal response, they were told to hold in their breath before each trial and then say "Aah!" clearly and loudly and with an abrupt attack. Their task was to synchronize the departure of their finger from the pressure pad with the onset of their voice. There were 15 trials, and each trial was initiated by the experimenter's saying "Go." The subjects were told to get ready to make responses when they heard the go signal, but they could start in their own time, as it was not a RT task. The subjects were told to hold their breath so as to be ready for voicing before each trial.

For the simple RT task, the subjects made finger, vocal, and both finger and vocal responses. They were instructed to respond as quickly as possible to the flash of the LED on the wall, by saying "Aah!," by releasing the pressure pad as before, or by performing both these responses, according to condition. There were 15 trials in each block with different response type, and each trial was initiated by the experimenter in the same way as in the synchronization task. The subjects were told to hold their breath in, so as to be ready for voicing before each trial. They were also told not to anticipate, and for this reason, the delay between the initiation and the flash was varied randomly.

For the coordination task, the subjects sat in front of the joystick assembly so that they could comfortably move the joystick from

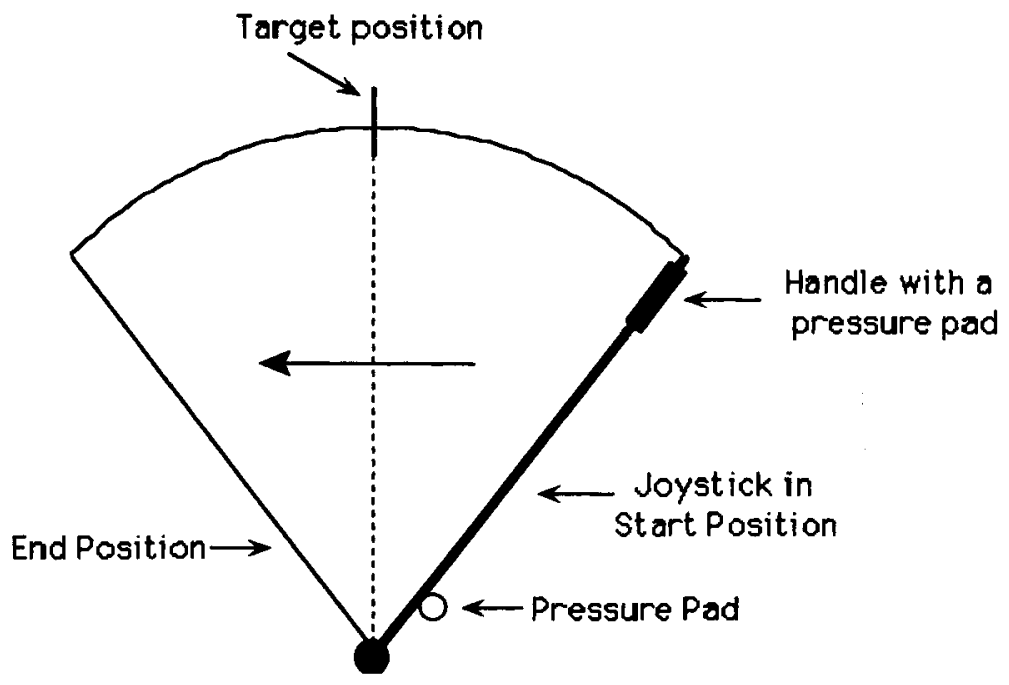

Figure 1. Schematic illustration of the apparatus, as viewed from the subject's position. 
side to side with their right arms. The subjects performed a target calibration before each block of experimental trials. In this calibration procedure, the subjects were asked to move the joystick to the target position and hold it there for $4 \mathrm{sec}$. The signal from the last $2 \mathrm{sec}$ was averaged and used to generate a subjective target position value. This procedure was important because the exact position of the subject with respect to the joystick could not be fixed, owing to factors such as individual differences in arm length. In order to minimize any inaccuracy owing to parallax effects, the subjects were instructed not to move their head or body for the duration of the block, once the calibration had been made.

After the calibration stage, the subjects were asked to make fluent movements of the joystick from right to left in the frontoparallel plane and to make a response at the moment the joystick passed in front of the target. They initiated the movement in their own time. One movement was made in each experimental trial, and there were 15 trials in each block. On a very few trials, no response was seen, and the trial was rerun at the end of the block. Before starting the task, the subjects practiced moving the joystick a few times without making any response. Full vision of the moving arm and of the apparatus was available throughout the experiment. No knowledge of results $(\mathrm{KR})$ was given at any stage.

As in the RT task, there were three blocks with different response types: finger, vocal, and both finger and vocal. For the finger response, the subjects pressed the pressure pad attached to the joystick handle with their index fingers before they started and kept a good pressure on it until they reached the target position, then released it sharply, just as the joystick passed through the target position. Similarly, the subjects made the vocal response by saying "Aah!" when passing through the target position. Again, the subjects were told to hold their breath in, so as to be ready for voicing before each trial. The subjects were told not to stop or slow down during a movement. The experimenter monitored the digitized joystick movement, paying particular attention to the portion around the target, and repeated the handful of trials that showed any intermittency.

The subjects were also encouraged to use a wide range of movement speeds. This was achieved by the experimenter's monitoring a histogram that showed the frequencies of different movement times and instructing subjects to move slower (or faster) on the next trial, so as to keep the histogram roughly uniform across a range of movement times common to all the subjects. This precaution was taken to prevent subjects from achieving perfect "coordination" by means of reproducing an identical arm trajectory on each trial and timing the secondary response from the onset of the unvarying arm trajectory. This kind of action need not involve any sharing of information between the primary arm movement and the response and does not constitute coordination in our sense of the term. In other words, forcing the subjects to modulate the speed of arm movement encouraged them to use information about the primary arm movement when timing the secondary response. In addition, monitoring arm movement speed ensured that the subjects made quantitatively comparable arm movements in all three response conditions.

\section{Results and Discussion}

A typical trace in the dual-response condition of the coordination task is given in Figure 2. The primary measure of coordination performance discussed in this paper

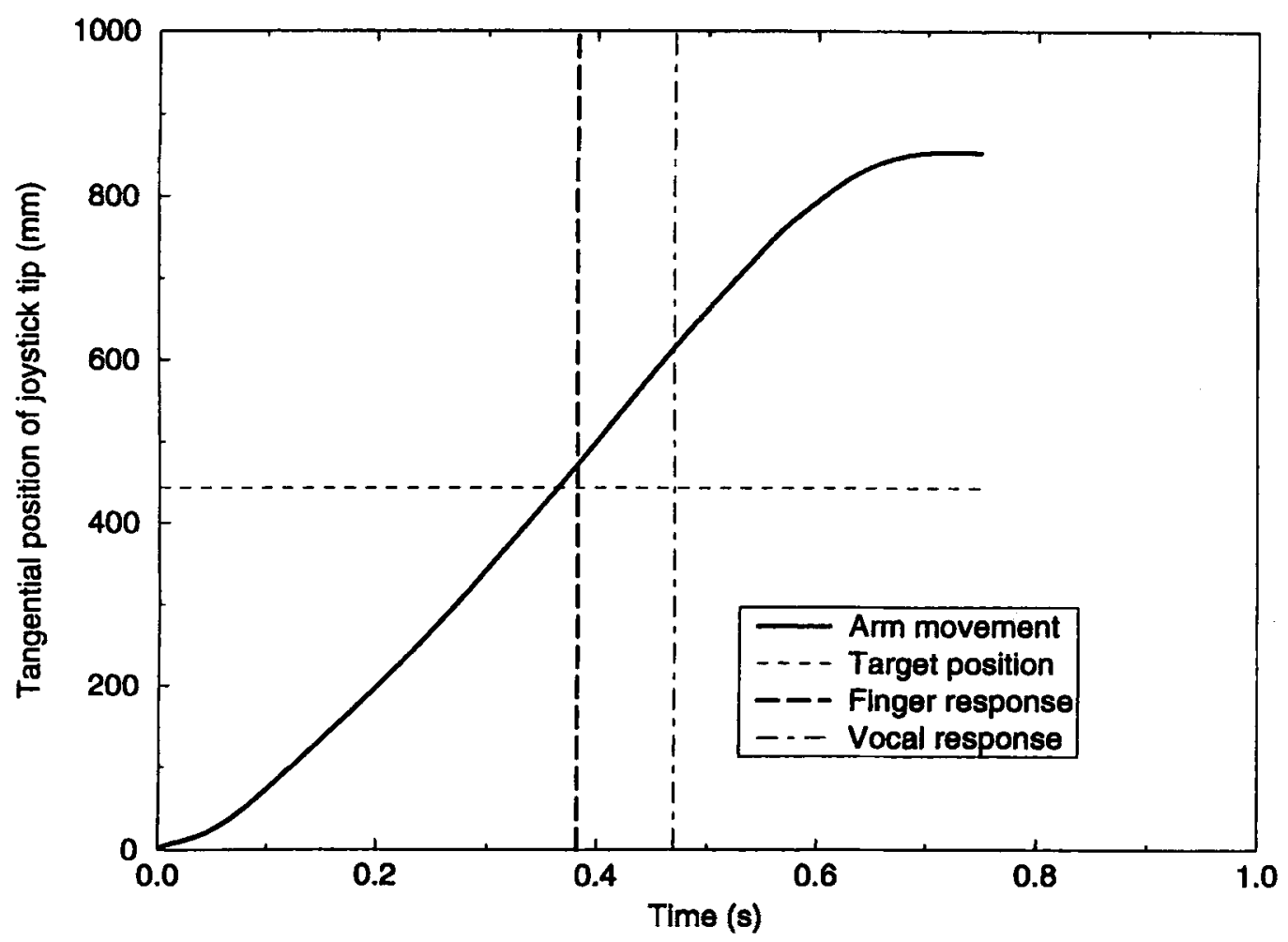

Figure 2. A typical trace in the dual-response condition of the coordination task. Note the smooth arm movement, without hesitancy or intermittency. The calibrated target position is shown by the dashed horizontal line. In this trial, both finger response (bold vertical dashed line) and vocal response (dot-dashed vertical line) were delayed, relative to passing the target, although the delay of the finger response is very small. The interval between the responses is the response onset asynchrony. 
is the delay of the responses. Delay in coordination corresponds to the time of the response on a given trial minus the time at which the joystick passed through the subjective target position on that trial. Negative delays thus correspond to anticipations. Response onset asynchronies (ROAs) were calculated when both finger and vocal responses occurred on a single trial - that is, for the synchronization task and for the dual-response conditions of the coordination and RT tasks. ROA was defined as the time of vocal response minus the time of finger response.

Delay analysis. A three-way repeated measures analysis of variance (ANOVA) with task (coordination or RT) $X$ response number (single or dual) $X$ response type (finger or vocal) as factors was conducted on the RT task data and the coordination task data. Synchronization data were omitted because, in the absence of any stimulus, the concept of delay does not apply. The main effect of task was, unsurprisingly, highly significant, with RTs being longer than coordination delays $[F(1,11)=259.09, p \leq .001]$. This result demonstrates that the subjects did not coordinate their movements by waiting until they visually perceived their arm passing the target and then initiating a reaction. The main effect of response type was also highly significant, with finger responses occurring, on average, $74 \mathrm{msec}$ earlier than vocal responses $[F(1,11)=115.27$, $p \leq .001]$. This is numerically close to the $82-\mathrm{msec}$ response effect observed by Haggard et al. (1999) in their experiments on the role of vision in coordinating responses. There was no significant main effect of response number $[F(1,11)=1.40, p=.2616]$. Two-way interactions of task $\times$ response number $[F(1,11)<1$, n.s. $]$ and of response number $\times$ response type $[F(1,11)<1$, n.s.] were not significant.

The two-way interaction of task $\times$ response type, however, was significant $[F(1,11)=10.85, p=.0072]$. This occurred because the difference between the finger and the vocal response delays was significantly larger in the coordination task (97-msec response effect) than in the simple RT task (52-msec response effect). That is, the response effect in coordination is $189 \%$ of that in the same subjects' RTs. We will return to this point in the discussion of this experiment.

Interestingly, the three-way interaction of task $\times$ response number $\times$ response type was significant $[F(1,11)=$ $10.87, p=.0071]$. This three-way interaction arose because finger responses were "attracted" toward the longer RTs of vocal responses in the dual-response condition of the RT task (see Figure 3b), whereas the vocal RTs themselves remained fairly stable. Indeed, post hoc testing of the RT condition showed response attraction (as measured by the response number $\times$ response type interaction) that approached statistical significance $[F(1,11)=4.72, p=$ $.0525]$. The coordination task, in contrast, shows a nonsignificant effect in the opposite direction: There is a small response repulsion in the dual-response condition. In the coordination task, the pattern of stable response types also seems to differ from that in the RT task: There is very little change in the finger response delays between the single- and dual-response conditions in the coordination task, whereas the vocal response shows a greater shift (see Figure 3a).

In conclusion, the effects of response type and response number on delays in coordination task are clearly different from the effects of those factors on RTs. In this sense, the information processing in the coordination cannot be considered to be a reaction to some internal stimulus generated by the moving arm, using the same circuits as reactions to external stimuli. Put another way, the circuits underlying coordination are qualitatively different from those underlying reactions.

We shall return to the nature of these circuits in the General Discussion section. For the moment, we shall discuss just three features of coordination. First, the increased response effect in coordination, relative to RT, suggests that the efferent account of response effects in coordination cannot be correct, since the efferent portion of the
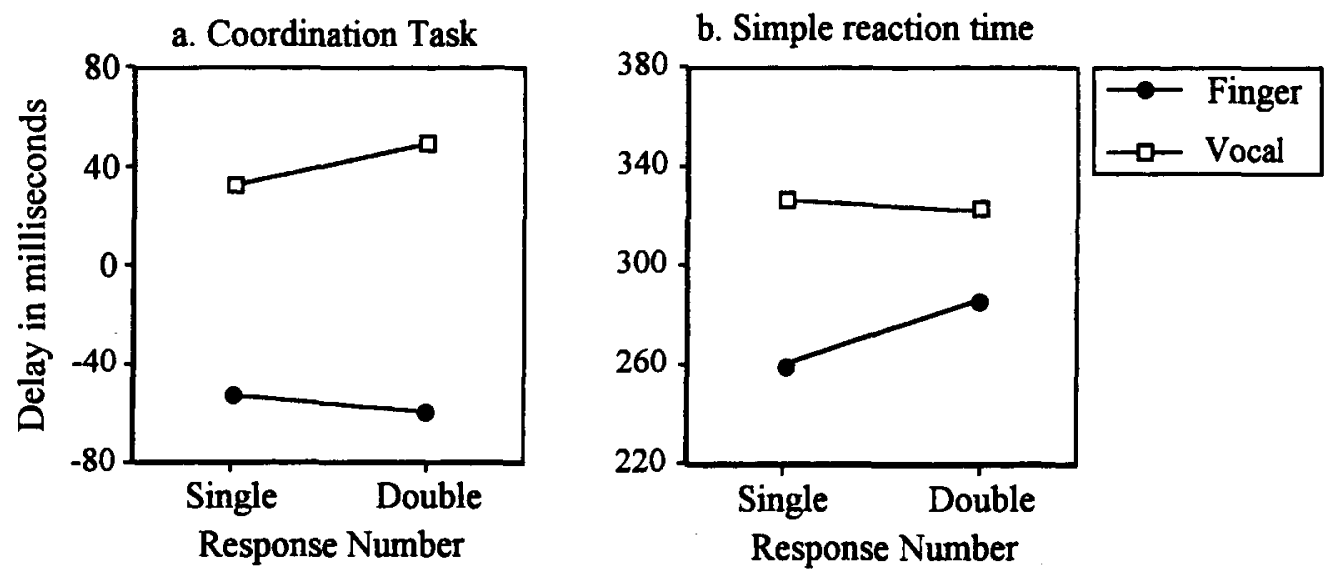

Figure 3. Mean delays in milliseconds for the coordination task (a) and the reaction time task (b). Note the similar scale but the different offsets in the two panels. Note also the difference in the direction of the two-way interactions in the two panels. 
circuit is the same in both tasks. Thus, the efferent account of response effects would predict equivalent response effects in any tasks requiring the same two responses. This was not observed in our data. Therefore, the response effect must occur prior to the efferent stage.

Second, the large response effect seen in the coordination task rules out an afferent explanation. The afferent information generated by the moving arm is presumably identical whatever coordinated response is made. Therefore, differences in the timing of responses cannot arise from differences in afferent information. Since both afferent and efferent accounts seem implausible, only a central locus for response effects remains, by elimination. That is, the central monitoring and processing of these stimuli generated by the moving arm must vary according to the response to be made. One model consistent with these findings would involve separate monitoring circuits for each response type. These response-specific monitoring circuits appear to have different temporal characteristics: The circuit leading to finger responses appears to be more predictive or anticipatory than the circuit leading to vocal responses.

Third, the absence of response attraction in dualresponse coordination, in contrast to its presence in RTs, deserves some comment. Response attraction has been observed between manual and oculomotor RTs (Bekkering, Adam, Vandenaarssen, Kingma, \& Whiting, 1995; Mather \& Fisk, 1985), typically in paradigms in which both responses are aimed at a single target location. Therefore, response attraction may arise from interactive enhancement of a representation of the target location. However, our RT task was not intrinsically spatial and did not require any aimed movement. Therefore, a more general tendency for parallel tasks to converge (Duncan, 1979) may underlie our result. More important, response attraction did not occur in the coordination task. If the working hypothesis of response-specific monitoring circuits is accepted, this result suggests that the two circuits studied here are informationally encapsulated and do not share information about the current arm position between each other. Since information is not shared across all these circuits, it seems plausible that some circuits will have better access than others to the internal stimuli generated by the moving limb and that this level of access will influence coordination performance. In this sense, these data support some form of availability hypothesis. Some putative models of information availability are considered in the General Discussion section.

Response onset asynchrony analysis. We analyzed the ROA between the finger and the vocal responses to investigate two representational hypotheses of response effects in coordination. ROAs were calculated for the dual-response conditions of the coordination and RT tasks and for the self-pace synchronization task. The Paillard-Fraisse hypothesis, generalized to the coordination task studied here, would predict an ROA equivalent to the difference in neural conduction times from the vocal cords to the brain and from the finger to the brain. The P-center hypothesis predicts an ROA equivalent to the difference between the physical response events instructed and measured and the (unknown) perceptual events by which the subjects internally represented those physical events. Crucially, however, both accounts predict equal ROAs in the self-paced synchronization task and in the coordination task. This is because, on a representational hypothesis, both tasks reduce to synchronizing internal representations.

The results showed a significant difference in ROA among the three tasks $[F(2,22)=240.43, p=.0001]$, with the mean ROA being significantly larger in the coordination task $(109.96 \mathrm{msec})$ than in both the RT task $(36.58 \mathrm{msec})$ and the self-paced synchronization task $(6.27 \mathrm{msec})$. Post hoc analysis revealed no significant difference between ROAs in the RT and synchronization tasks.

\section{EXPERIMENT 2}

The results from Experiment 1 suggested that the response effect in the coordination task is not solely due to differences in operation of the efferent systems, but to an information availability effect, whereby the circuit underlying finger responses has more predictive information about the primary arm movement than does the circuit for vocal responses. However, if there is a specialized circuit for coordination of finger responses, Experiment 1 cannot exclude the possibility that response effects arise because vocal responses require mediation by awareness of internal stimuli generated by the moving arm, whereas finger responses are not mediated by such awareness (the awareness hypothesis mentioned in the introduction). Because the operation of awareness requires additional time, differential mediation by awareness for the two response conditions would produce a response effect.

A natural way of deciding between availability and awareness hypotheses of response effects involves comparing delays between a condition in which the finger response is made by the same hand that moves the joystick and a condition in which the response is made by the contralateral hand. An availability hypothesis predicts a difference between these conditions, since it is presumed that responding with the contralateral hand involves an additional stage in transferring information, presumably via the corpus callosum from one sensory cortex to the contralateral motor cortex. This stage should cause an additional delay in the contralateral coordinated response. The awareness hypothesis predicts no difference between these conditions, since motor responses in either hemisphere are presumably equally unconscious. The purpose of the second experiment was to investigate these hypotheses, using a coordination task.

\section{Method}

Experiment 2 used a $2 \times 3$ factorial design. The factors were (1) whether the subject used their right or left arm to move the joy- 
stick (arm factor), and (2) whether the response was made with the right index finger, the left index finger, or vocally (response type factor). Group was a between-subjects factor; subjects were randomly assigned to one of four groups in order to counterbalance the order of conditions. Groups A and B made the left arm movements (L) before the right arm movements $(R)$, whereas Groups $C$ and $D$ used the opposite order. Groups $A$ and $C$ made the left finger responses (L) before the right ones $(R)$, whereas this was reversed for Groups B and D. All the groups made the vocal responses first, as Experiment 1 had shown that there was no order effect between finger and vocal responses. Arm and response type were within-subjects factors. Again, delay in milliseconds was used as the dependent variable. Data acquisition was the same as that in Experiment 1.

Subjects. Twelve normal subjects, 6 male and 6 female, participated in this experiment. All of them were proven to be right-handed by the Edinburgh Handedness Inventory (Oldfield, 1971). Their ages ranged from 24 to 45 years, with an average age of 30 years. Two of them had previously participated in Experiment 1, but all were naive as to the purpose of the experiment.

Procedure. Instructions were given before each block and were similar to those in the coordination task in Experiment 1 . The only differences were that subjects moved the joystick from left to right, using their left arm, in half of the blocks and right to left, using the right arm, in the other half. This was done to ensure that the movements made by the two arms were congruent. When the subjects used different hands for the primary movement and the finger response, the finger response was made by releasing a pressure pad attached to a wooden block, placed on their knees. Otherwise they released a pressure pad mounted on the joystick, as in Experiment 1. There were 20 trials in each of the six blocks, but the first 5 trials in each block were treated as practice and were excluded from analysis. We included these additional practice trials to allow subjects to achieve more fluent primary arm movements with the nondominant left hand. A separate calibration was used to establish the subjective target position prior to each block, and this value was used to calculate delays for that block's trials, as in Experiment 1.

\section{Results and Discussion}

Delay analysis. A three-way ANOVA, treating group as a between-subjects factor and arm and response type as within-subjects factors, was conducted on the delay data. Delay was calculated in the same way as that in Experiment 1.
The main effect of response type was significant $[F(2,16)=7.962, p=.004]$. Post hoc analysis, using Tukey's HSD test, showed that vocal responses $(22.408 \mathrm{msec})$ were significantly slower than right-finger responses $(-26.762 \mathrm{msec})$ or left-finger responses $(-34.190 \mathrm{msec})$. Interestingly, the main effect of arm was not significant $[F(1,8)<1$, n.s.), thus precluding any difference in the quality or quantity of information produced by moving the two arms. The difference between left- and right-finger responses was not significant, thus excluding the hypothesis that one finger has superior coordination performance. The arm $\times$ response interaction was, however, significant $[F(2,16)=5.889, p=.002]$. This interaction is clearly due to the relationship between arms and the finger responses and does not include the vocal response (Figure 4). This crossover interaction suggests that an availability process of some kind must be at work.

Finally, the size of the constant errors deserves some comment. Responses made with the ipsilateral hand were more anticipatory than those made contralaterally. In a strict sense, therefore, they were less accurate. However, we are reluctant to say that ipsilateral coordination is generally worse than contralateral coordination on the basis of these data, because the task studied here is highly artificial in one important sense. Specifically, our subjects had no KR at any stage. This situation almost never arises in everyday actions such as throwing, which typically afford one or more forms of KR (e.g., where the ball goes when we throw it) that we can use to adjust our coordination. Aschersleben (in press) found that the anticipatory constant errors seen in synchronization with external stimuli disappear with extensive practice, as long as $\mathrm{KR}$ is given. Cordo et al. (1994) studied a task similar to that reported here, but one in which $K R$ was given on every trial. They observed much smaller errors than those in our study, and these were not always anticipatory.

Absence of $\mathrm{KR}$ allows us to study the intrinsic temporal characteristics of the circuits involved in coordinated responses, without additional effects caused by

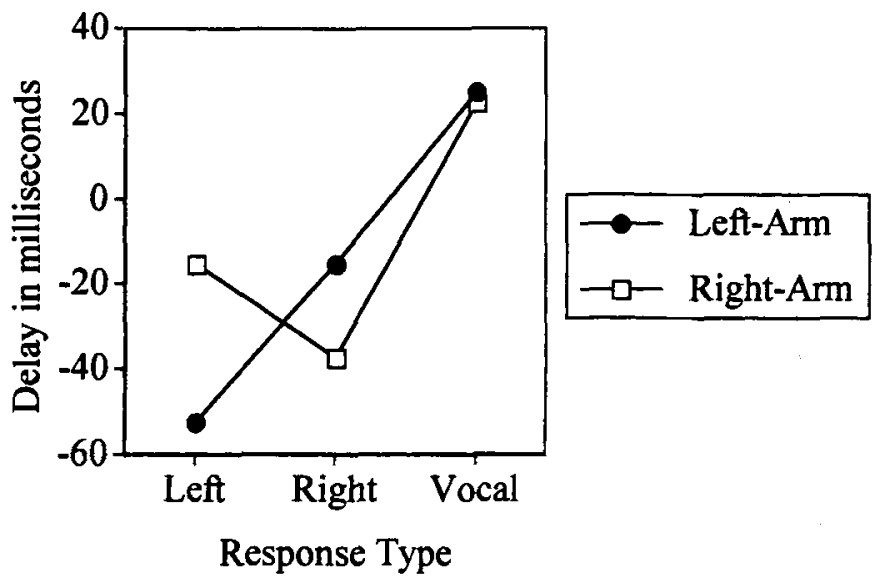

Figure 4. Mean delays in milliseconds for coordinated responses in Experiment 2. 
learning the task. Anticipation appears to be a consistent feature of these circuits, and it is more evident for ipsilateral than for contralateral responses. We speculate that this reflects a greater element of prediction in coordinated actions restricted to one side of the body.

Variable error of delays. Another analysis was conducted on standard deviation $(S D)$ data in order to focus on the variable error-that is, the degree of scatter in the timing of coordinated responses. Variable error is particularly relevant to the information availability hypothesis, since superior information transmission between the arm and the center making the coordinated responses should lead to more consistent coordination performance. This corresponds to decreased random errors in coordination. The $S D$ of the 15 experimental trials by each subject in each condition was therefore calculated and subjected to the same ANOVA as the delay data. Results showed that none of the main effects or interactions of group, of arm, and of response type were significant. Nevertheless, the two-way interaction of arm $\times$ response type showed a trend toward statistical significance $[F(2,16)=2.479, p=$ $.115]$. Inspection of the data (see Table 1) showed that this was caused by relatively low $S D$ s in the R-R condition.

In order to have a better view of this interaction of arm $X$ response type, suggested in the above analysis, a robust modification of the sample variance ratio test, proposed by Box and Andersen (1955), was used. This test compares the variance of two samples, adjusting the degrees of freedom of a standard $F$ ratio, to compensate for bias introduced by the possible nonnormality of the data. This approach allowed us to perform pairwise comparisons at $p=.05$ of each subject's variable error for all four combinations of finger response and arm movement factors (vocal responses were excluded from this analysis). We then applied a binomial probability test to the number of subjects who showed significant differences for each of these comparisons. The binomial test showed that the number of subjects with significant differences in variable error was above the .05 significance level for only two of these pairwise comparisons, discussed below.

First, right arm + right finger responses had lower variable errors than right arm + left finger responses for 11 of the 12 subjects, with three of these differences being significant in the Box-Andersen test. The binomial probabilities of this pattern of orderings $[B(12,0.5,1)=.0032]$ and significances $[B(12,0.05,3)=.020]$ are themselves significant. Is this effect due to better information sharing between the right arm and the right finger response

Table 1

Variable Error (in Milliseconds) of Delays in Experiment 2

\begin{tabular}{cccc} 
& \multicolumn{3}{c}{ Response type } \\
\cline { 3 - 4 } Movement Type & Left & Right & Vocal \\
\hline Left arm & 60.46 & 66.42 & 59.86 \\
Right arm & 71.51 & 51.94 & 57.31 \\
\hline
\end{tabular}

Note-The value is the mean across subjects of the standard deviation across trials in each condition. conditions, or merely to greater precision and consistency in the production of right finger responses? The latter possibility was investigated by comparing right arm + right finger responses with left arm + right finger responses. If consistency of right finger responses determines variable error, these conditions should not differ. We found, however, that right arm + right finger responses had lower variable errors than left arm + right finger responses for 10 of the 12 subjects, with four of these differences being significant in the Box-Andersen test. The binomial probabilities of this pattern of directions $[B(12,0.5,2)=.0192]$ and significances $[B(12,0.05,4)=.002]$ are themselves significant. Taken together, these post hoc tests suggested that the low $S D$ in the right arm + right finger response condition is not due to superior information obtained from right arm movement (which would have produced comparably low variable errors in the right arm + left finger response condition) or to superior right finger responses (which would have produced comparably low variable errors in the left arm + right finger response condition), but to the combination of these two factors. It thus represents an availability or compatibility effect. Interestingly, we did not observe any comparable availability effect for the left hand, perhaps because all our subjects were right-handed. We speculate that an inverse effect to that reported here might, nevertheless, be present in left-handed subjects.

This result is based on an exploratory post hoc analysis of our own particular subjects' data and does not necessarily generalize to the population. However, we report it in order to highlight the possibility that the availability of information may affect variable error as well as constant error. Given the ambiguity and lability of constant errors in the absence of KR (see above), we are developing the use of variable error as an index of coordination in our current research.

\section{GENERAL DISCUSSION}

In both Experiment 1 and Experiment 2, we have found large response effects, reflected in the different delays of finger and vocal responses in a coordination task in which these responses are made as the arm passes through a target location. Finger responses were constantly anticipatory, whereas vocal responses were delayed. The comparison with a simple RT task in Experiment 1 suggested that the response effect cannot be entirely attributed to the difference between efferent systems, but also involves an availability effect between the effectors and the internal stimuli generated by arm movement. In particular, there seemed to be a specialized S-R pathway that facilitates finger responses in coordination. Since finger responses are typically anticipatory, this pathway must be predictive. The results from Experiment 2 demonstrated that this special circuit can share information across two sides of the body, but only at the cost of a significant additional delay. 
There are four possible explanations for this response effect. The first and simplest is an efferent explanation (Anson, 1982): The motor delay between a central command and a finger movement could be shorter than the motor delay between a central command and the acoustic signal of a vocal response. The second explanation invokes a P-center hypothesis (Aschersleben \& Prinz, 1995): Both finger and vocal responses are extended events, whose perceptual center need not coincide with their physical onset. If, for example, vocal responses are perceived to occur much earlier than their physical onset, whereas finger responses are perceived to occur much later than their physical onset, these representational differences could explain the response effects we have observed. A third explanation, based on a generalization of Castiello et al.'s (1991) awareness effect, suggests that the two responses may involve awareness to different extents and that mediation by awareness may bring an additional delay for vocal responses. The fourth explanation of the response effects invokes an S-R compatibility effect whereby some response centers have better access than others to the internal stimuli generated by the moving arm.

Our data are consistent with the availability explanation of response effects, but not with the other three. The efferent explanation can be ruled out by comparing coordination with RT performance. As the efferent portion of the response is similar in the two tasks, the efferent hypothesis predicts equal response effects. We found significantly larger response effects in the coordination task, suggesting that efferent mechanisms cannot fully explain our coordination findings.

The P-center and Paillard-Fraisse hypotheses can likewise be ruled out by comparing the synchronization and coordination tasks. The P-center hypothesis would explain the large ROA in the coordination task by propos- ing that the perceived time of a finger response might lag the physical event of the finger moving off the pressure pad, whereas the perceived time of a vocal response might not be the onset of laryngeal movement but some earlier event. There are both theoretical and empirical reasons for rejecting this view. First, it is counterintuitive that the release of the pressure pad, which marks the culmination of the contraction of the finger extensions, should be perceived to happen after it does: The gradual change in force makes an early P-center more plausible than a late one. Second, evidence from speech studies (see the introduction) suggests that speech P-centers lag the onset of acoustic energy, although our data would require an effect in the opposite direction. Third, the P-center interpretation of our coordination data would predict an ROA in the synchronization task that is equal in size to that seen in the coordination task. This is because the P-center hypothesis states that the synchronization task involves synchronizing perceptual centers, not physical events. Our data shows that response effects in coordination are an order of magnitude greater than those in synchronization. Finally, the possibility that response effects reflect differential awareness of stimuli from the moving arm, comparable with the effects seen by Castiello et al. (1991), is harder to exclude. This explanation would attribute the delay of vocal responses, relative to finger responses, to the mediating role of awareness in the former but not in the latter. However, our data reveal several aspects of response effects that cannot be explained in this way. First, the response effect is larger in the coordination task than in the RT task. The putative explanation that the larger response effect in coordination reflects a greater level of awareness of internal stimuli generated by the moving arm than of external stimuli flies in the face of the common intuition that we have little conscious knowledge about the progress of our own

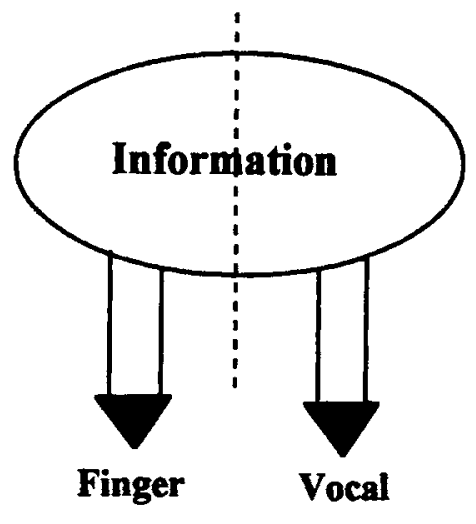

$$
\begin{aligned}
& \text { a. Encapsulated } \\
& \text { information framework }
\end{aligned}
$$

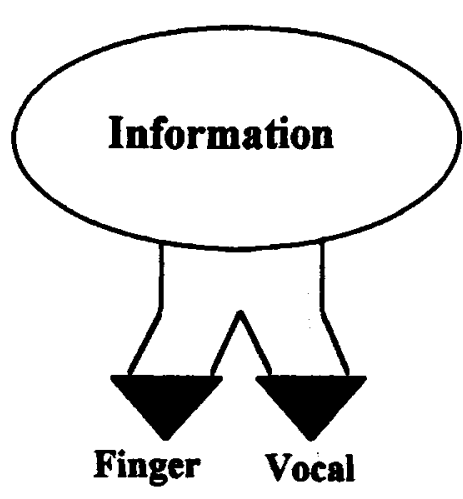

b. Mutual access

Figure 5. Schematic illustrating the different arrangements of informationally encapsulated (a) and mutually accessible (b) response circuits. In informationally encapsulated circuits, information used in generating one response cannot be used to improve another response. In mutually accessible circuits, information is shared between response centers until the final efferent part of the circuit begins. 
actions. Second, we observed significant differences in response timing between the left and the right finger responses in Experiment 2. Since it seems implausible to argue that awareness is an attribute of one finger's response but not of another's, this suggests that a part of the response effect cannot be due to delays associated with awareness.

This leaves the availability hypothesis as the most plausible explanation of response effects. There are two possible reasons why information about the internal stimuli generated by the moving arm might be differentially available to response centers. First, there may be differences in the amount or quality of stimulus information which response centers can access. Second, information may be equally available but may require additional translation stages before it can be used by some response centers. The translation process may require significant computation and take significant time, with concomitant effects on performance.

\section{Can Information be Shared Between Response Pathways?}

A view that opposes the concept of shared information predicts that the pathways subserving those two responses are separate within the CNS and informationally encapsulated (Figure 5a). Consequently, the responses would remain the same in the dual-response condition, since each pathway is independent from the other. Alternatively, if the response pathways for those two responses are mutually accessible, the pathway with poorer information about the primary movement can nevertheless produce an appropriate response by accessing information in the other, "dominant" response pathway (Figure 5b). In this case, response attraction would be observed (Duncan, 1979).

The findings from the coordination task in our Experiment 1 supported the information encapsulation view, because the timing of the two responses was comparable in single- and dual-task conditions. If there were some mutual access between the two response pathways, the vocal response could have gained more up-to-date information derived from the response pathway for the finger responses, which would have led to a reduced delay in vocal responses. In the RT task, in contrast, the mutual access theory was supported, because the vocal response attracted the finger response, even though this led to increased finger RTs. These findings suggest that the information processing in the coordinated movement task is qualitatively different from that in the simple RT tasks: Movement coordination appears to involve informationally encapsulated circuits, whereas RT tasks appear to involve pooling of information.

In our Experiment 2, we showed that the anticipatory quality of finger responses was reduced when the response was made contralaterally to the moving arm. There appears, then, to be a delaying effect and loss of precision, at least in our subjects, associated with sharing information across both sides of the body. We have not, however, looked at the condition in which both fingers make a response when the arm passes through the target. We plan to do this in future research, to investigate whether the circuits subserving coordination on the two sides of the body are informationally encapsulated from each other. It may be that information can be pooled between circuits as long as the responses those circuits produce are similar, even if the effectors are represented in different hemispheres.

\section{REFERENCES}

Anson, J. G. (1982). Memory drum theory: Alternative tests and explanations for the complexity effects on simple reaction time. Journal of Motor Behavior, 14, 228-246.

AsCHERSLEBEN, G. (1994). Afferent Information und die Synchronisation von Ereignissen [Afferent information and the synchronization of events]. Frankfurt: Peter Lang

AsChERSLEBEN, G. (in press). Knowledge of results and the timing of actions. Journal of Motor Behavior.

Aschersleben, G., \& Prinz, W. (1995). Synchronizing actions with events: The role of sensory information. Perception \& Psychophysics, 57, 305-317.

Barber, P. (1988). Applied cognitive psychology: An information processing framework. London: Routledge.

Bekkering, H., Adam, J. J., Vandenaarssen, A., Kingma, H., \& WhitiNG, H. T. A. (1995). Interference between saccadic eye and goal-directed hand movements. Experimental Brain Research, 106, $475-484$

Box, G. E. P. \& Andersen, S. L. (1955). Permutation theory in the derivation of robust criteria and the study of departures from assumption. Journal of Royal Statistical Society: Series B, 17, 1-26.

Bridgeman, B., Peery, S., \& ANAND, S. (1997). Interaction of cognitive and sensorimotor maps of visual space. Perception \& Psychophysics, 59, 456-469.

Castiello, U., \& Jeannerod, M. (1991). Measuring time to awareness. NeuroReport, 2, 797-800.

Castiello, U., Paulignan, Y., \& Jeannerod, M. (1991). Temporal dissociation of motor responses and subjective awareness. Brain, 114, 2639-2655

Cordo, P. J. (1990). Kinaesthetic control of a multijoint movement sequence. Journal of Neurophysiology, 63, 161-172.

Cordo, P. J., Carlton, L., Bevan, I., Carlton, M., \& Kerr, G. K. (1994). Proprioceptive coordination of movement sequences: Role of velocity and position information. Journal of Neurophysiology, 71, 1848-1861.

CORDo, P. J., \& Flanders, M. (1989). Sensory control of target acquisition. Trends in Neurosciences, 12, 110-116.

Duncan, J. (1979). Divided attention: The whole is more than the sum of its parts. Journal of Experimental Psychology: Human Perception \& Performance, 5, 216-228.

DunLoP, K. (1910). Reactions on rhythmic stimuli, with attempt to synchronize. Psychological Review, 17, 399-416.

Fraisse, P. (1974). Cues in sensori-motor synchronization. In L. E. Scheving, F. Halberg, \& J. E. Pauly (Eds.), Chronobiology (pp. 517. 522). Tokyo: Agaku Shoin.

GANDEVIA, S. C. (1987). Roles for perceived voluntary motor commands in motor control. Trends in Neurosciences, 10, 81-85.

Gandevia, S. C., \& Burke, D. (1992). Does the nervous system depend on kinaesthetic information to control natural limb movements? Behavioral \& Brain Sciences, 15, 614-632.

Goodale, M. A., Milner, A. D., Jakobson, L. S., \& Carey, D. P. (1991a). A neurological dissociation between perceiving objects and grasping them. Nature, 349, 154-156.

Goodale, M. A., Milner, A. D., Jakobson, L. S., \& Carey, D. P. (1991b), Object awareness. Nature, 352, 202.

Goodale, M. A., Pelisson, D., \& Prablanc, C. (1986). Large adjustments in visually guided reaching do not depend on vision of the hand or perception of target displacement. Nature, 320, 748-750.

GreENWALD, A. G. (1972). On doing two things at once: Time sharing 
as a function of ideomotor compatibility. Journal of Experimental Psychology, 94, 52-57.

Greenwald, A. G., \& Shulman H. G. (1973). On doing two things at once: II. Elimination of the psychological refractory period effect. Journal of Experimental Psychology, 101, 70-76.

haggard, P., Lyon, I., Bradbury, C., \& Azuma, R. (1999). Coordination of a discrete response with a specified position during arm movement. Manuscript submitted for publication.

Henry, F. M., \& Rogers, D. E. (1960). Increased response latency for complicated movements and a memory drum theory of neuromotor reaction. Research Quarterly, 31, 448-458.

JEANNEROD, M. (1981). Intersegmental coordination during reaching at natural visual objects. In J. [B.] Long \& A. [D.] Baddeley (Eds.), Attention and performance $I X$ (pp. 153-169). Hillsdale, NJ: Erlbaum.

LEONARD, J. A. (1959). Tactual choice reactions: I. Quarterly Journal of Experimental Psychology, 12, 76-83.

MATHER, J. A., \& Fisk, J. D. (I985). Orienting to targets by looking and pointing - parallels and interactions in ocular and manual performance. Quarterly Journal of Experimental Psychology, 37A, 315-338.

Morton, J., Marcus, S. M., \& Frankish, C. R. (1976). Perceptual centers (P-centers). Psychological Review, 83, 405-408.
OLDFIELD, R. C. (1971). The assessment and analysis of handedness: The Edinburgh Inventory. Neuropsychologia, 9, 97-113.

Proctor, R. W. \& ReEve, T. G. (EDS.) (1990). Stimulus-response compatibility: An integrated perspective. Amsterdam: North-Holland.

SchMid, R. A. (1988). Motor control and learning: A behavioral emphasis (2nd ed.). Champaign, IL: Human Kinetics.

STEIN, J. F., \& GLICKSTEIN, M. (1992). Role of the cerebellum in visual guidance of movement. Physiological Reviews, 72, 967-1017.

Vos, P. G., \& HelsPER, E. L. (1992). Tracking simple rhythms: On-beat versus off-beat performance. In F. Macar, V. Pouthas, \& W. J. Friedman (Eds.), Time, action and cognition: Towards bridging the gap (Vol. 66, pp. 287-299). Dortrecht: Kluwer.

Vos, P. G., Mates, J., \& von Kruysbergen, N. W. (1995). The perceptual center of a stimulus as the cue for synchronization to a metronome: Evidence from asynchronies. Quarterly Journal of Experimental Psychology, 48A, 1024-1040.

WEISKRANTZ, L. (1974). Visual capacity in the hemianopic field following restricted occipital ablation. Brain, 97, 709-729.

(Manuscript received February 18, 1997; revision accepted for publication March 25, 1998.) 